\title{
Is Hookah Smoking a Better Alternative in Reducing Stress?
}

\author{
Mala Thakur* \\ School of Medicine, Xavier University, Aruba
}

*Corresponding author: Mala Thakur, Xavier University School of Medicine, Oranjestad, Aruba.

To Cite This Article: Mala Thakur, Is Hookah Smoking a Better Alternative in Reducing Stress?. Am J Biomed Sci \& Res. 2020 - 6(6). AJBSR. MS.ID.001095. DOI: 10.34297/AJBSR.2020.06.001095.

Received: 眥 December 30, 2019; Published: 漹 January 16, 2020

\begin{abstract}
Growing popularity worldwide, Hookah has become a new trend that many individuals are seeking as an alternative to smoking cigarettes. These waterpipe devices are attracting many individuals, especially the younger generation with its easy accessibility and assorted flavors. Everyday different types of E-cigarettes are becoming available in the market due to the popular demand. Across the world, many individuals are turning to this alternative method of cigarette smoking for a sense of stress relief. In this mini review, the correlation between hookah smoking and its effects on reducing stress were reviewed.
\end{abstract}

\section{Introduction}

Hookah, a waterpipe device used in many cultures with different names (e.g., shisha, narghile), is a [1] traditional centuries old tobacco used method that is becoming globally popular [2]. Hookah smoking is a popular trend in the United States amongst young adults and teenagers. This new form of smoking gives the individual a false sense of a safer alternative to smoking a cigarette. Although people who smoke Hookah may think it is less harmful when compared to a cigarette, it actually imposes the same health risks as cigarette smoking [2,3]. As hookah lounges are on a rise due to popular demand, so are the health risks. Despite these risks, individuals still continue to ignore the warning signs and continue to inhale these carcinogens due to the simplicity that it helps alleviate stress.

\section{Methods}

The data presented here were gathered via PubMed, Google Scholar, and Research Gate in attempts to collect peer reviewed articles for reference regarding waterpipe smoking's effects on stress. The information presented is factual and up to date as guaranteed by the authors. The purpose of this research is to provide further insight on Hookah smoking and its alleviation on stress.

\section{Discussion: Pathogeneisis in Reduction of Stress}

Everyday humans are exposed to stressful situations which cause an alteration in homeostasis within the human body. Stress is the biological response that is evoked by an intrinsic or extrinsic stimulus [4]. This causes a toll in the human body, especially on mental health. The hippocampus, amygdala, and prefrontal cortex are areas of the brain which are known to contain high levels of glucocorticoid receptors since they are controlled by the hypothalamic-pituitary- adrenal axis [5]. This axis is the central stress response system that gets activated by releasing the corticotropin releasing hormones in response to when our body releases increase levels of epinephrine and norepinephrine during stress.

Hookah flavors consists of nicotine, an amine that is an addictive agent which is added to tobacco products. Once nicotine is inhaled in the form of smoke, it is immediately entered into the blood stream. Nicotine takes about 2-8 seconds from the time of inhalation to cross the blood brain barrier [6]. Within the brain after nicotine enters the blood brain barrier, it binds to nicotinic cholinergic receptors ( $\mathrm{nAChRs)}$ which causes the production of neurotransmitter release. Dopamine is the main neurotransmitter which is released and is responsible in making an individual feel good. This is one of the many reasons how a person may get addicted to smoking. The average amount of nicotine present in the hookah flavors is about $3.4 \mathrm{mg} / \mathrm{g}$ [7]. This average is low compared to the daily intake of cigarettes, which happens to consist of 37.6 $\mathrm{mg} / \mathrm{g}$ of nicotine [8]. Stress relief after inhalation comes from the nicotine that is present in hookah as well as any tobacco product. 


\section{Conclusion}

Hookah flavored tobacco consists of nicotine which helps in the release of dopamine once inhaled. This release is what causes an individual to feel good, or a sense of calmness when stressed. Hookah smoking is a safer alternative in reducing stress when compared to cigarette smoking in regard to the nicotine content. Hookah's flavored tobacco contains far less nicotine than cigarettes, and individuals may not become as addicted to hookah when compared to cigarette smoking. The dangerous health risks still remain the same in both cases regardless of the amount of nicotine content present.

\section{References}

1. (2007) American Lung Association. An Emerging Deadly Trend: Waterpipe Tobacco UseCdc-pdf External. [PDF $222 \mathrm{~KB}$ ] Washington: American Lung Association, USA.
2. Maziak W (2013) The waterpipe: an emerging global risk for cancer. Cancer Epidemiol. 37(1): 1-4.

3. (2011) American Lung Association. Hookah Smoking: A Growing Threat to Public Health Issue Brief. Smokefree Communities Project.

4. Yaribeygi, H Panahi, Y Sahraei, H Johnston, TP Sahebkar A, et al. (2017) The impact of stress on body function: A review. 16: 1057-1072.

5. Lucassen PJ, Pruessner J, Sousa N, Almeida, Anne Marie Van Dam, et al. (2013) Neuropathology of stress. Acta Neuropathologica 127(1): 109135.

6. Widysanto A, Combest FE, Saadabadi A (2019) Nicotine Addiction. In: StatPearls [Internet], Indonesia.

7. Shafagoj YA, Mohammed FI, Hadidi KA (2002) Hubble-bubble (water pipe) smoking: levels of nicotine and cotinine in plasma, saliva and urine. Int. Journal of Clinical Pharmacology and Therapeutics, 40(6): 249-255.

8. Benowitz NL, Jacob P (1984) Daily intake of nicotine during cigarette smoking. Clin Pharmacol Ther 35(4): 499-504. 